\title{
Correction to: Metabolic features of recurrent major depressive disorder in remission, and the risk of future recurrence
}

Roel J. T. Mocking, Jane C. Naviaux, Kefeng Li, Lin Wang, Jonathan M. Monk, A. Taylor Bright, Caroline A. Figueroa (D, Aart H. Schene, Henricus G. Ruhé, Johanna Assies and Robert K. Naviaux (1)

\section{Correction to: Translational Psychiatry \\ https://doi.org/10.1038/s41398-020-01182-w \\ published online 11 Jan 2021}

The original version of this article unfortunately contained a mistake. The pdf version of the article was missing the correct Fig. 4. Instead, the pdf used the Fig. 4 title and legend on page 10 for Fig. 3 panels I-P. The Venn diagram in the true Fig. 4 has been dropped from the pdf. The correct figure can be found below. We apologize for the mistake. The original article has been corrected.

Published online: 08 February 2021

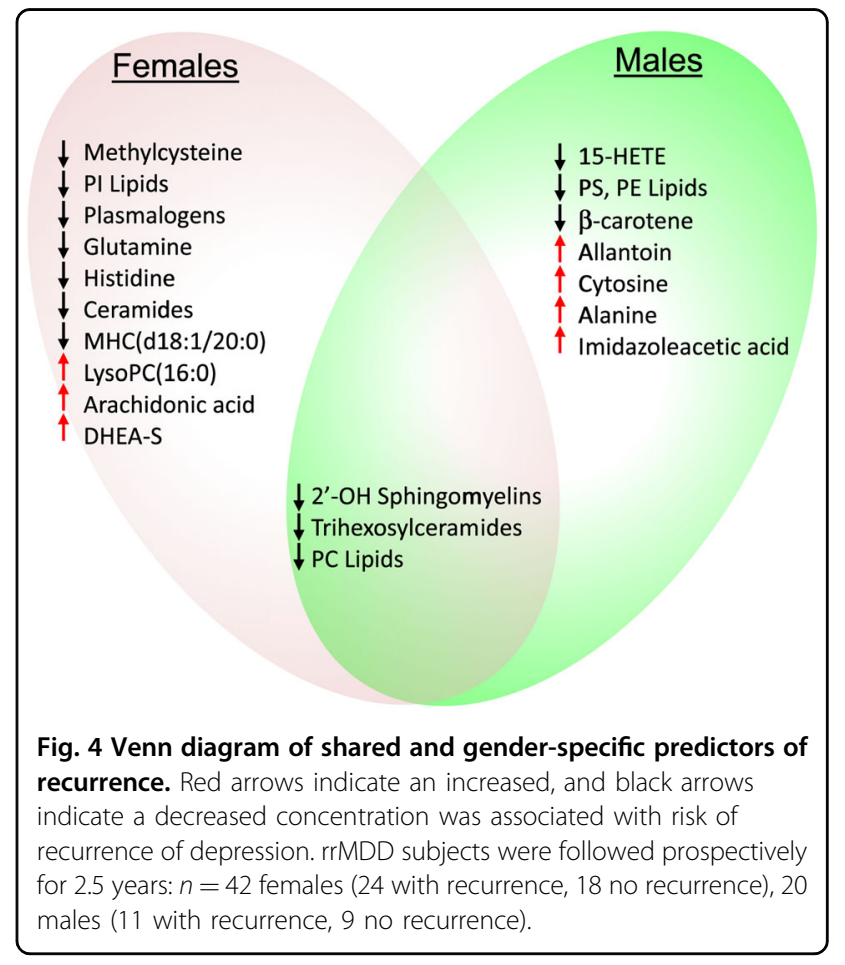

